\title{
Traditional paintings and the digital medium
}

\author{
Bruno Barberi Gnecco \\ Corollarium Tecnologia \\ Itapecerica da Serra, Brazil \\ brunobg@corollarium.com
}

\section{Marcelo de Paiva Guimarães}

Universidade Federal de São Paulo/Programa de Pósgraduação da Faculdade Campo Limpo Paulista

São Paulo, Brazil

marcelodepaiva@gmail.com

\author{
Diego Roberto Colombo Dias \\ Universidade Federal de São Carlos \\ Departamento de Ciência da Computação \\ São Carlos, Brazil \\ diegocolombo.dias@gmail.com
}

\begin{abstract}
We describe in this paper the experience of bringing a hyper-realistic painting by the Brazilian plastic artist Sônia Menna Barreto into the digital world, taking advantage of its interactive and animated possibilities to bring the work to life. To do that, we developed software to visualize the modeled painting in a multiprojection environment (mini CAVE), in desktops, in digital TV devices and even smartphones and tablets. We also describe how natural interaction and navigation through the digital painting was accomplished by using Wii-remote and Kinect devices.
\end{abstract}

Key-words — art, surrealism, virtual reality

\section{INTRODUCTION}

Digital art has been growing amazingly over the last few years, thanks to cheaper technologies and new software. Many new coding languages and frameworks have been developed with art in mind, making it easier for artists with no technological background to learn and to use them. New devices, such as cameras and touch sensitive surfaces, are now cheap and ubiquitous. There is still, however, a great chasm between traditional and digital art; we need to find out in which ways they can be used to complement each other, instead of considering them different manifestations that are completely unrelated.

If we can bridge this chasm we open new possibilities. We can exploit the best characteristics of these tools and make art even more involving. Technology can enrich traditional art, not only by providing the art viewer with more information (text, images, videos, which are helpful to understand the artwork, its theme, technique, style, history etc.), but much more: the viewer can interact and appreciate the work at a whole new level. Art is fragile and we cannot have people touching the works, or even getting too close to them; altering an original work is unthinkable. But in the digital world we have these possibilities: the viewer can interact, learn, explore, even modify the artwork.

We explore in this paper an application that bridged this chasm, starting from a traditional painting and leading to a digital artwork, interactive and immersive. We explore several forms of viewing and interacting with this work. The original painting was painted by Sônia Menna Barreto, a well-known Brazilian artist. Figure 1 shows the painting we chose, "Torre de Papel" ("Paper Tower"), in its original form.

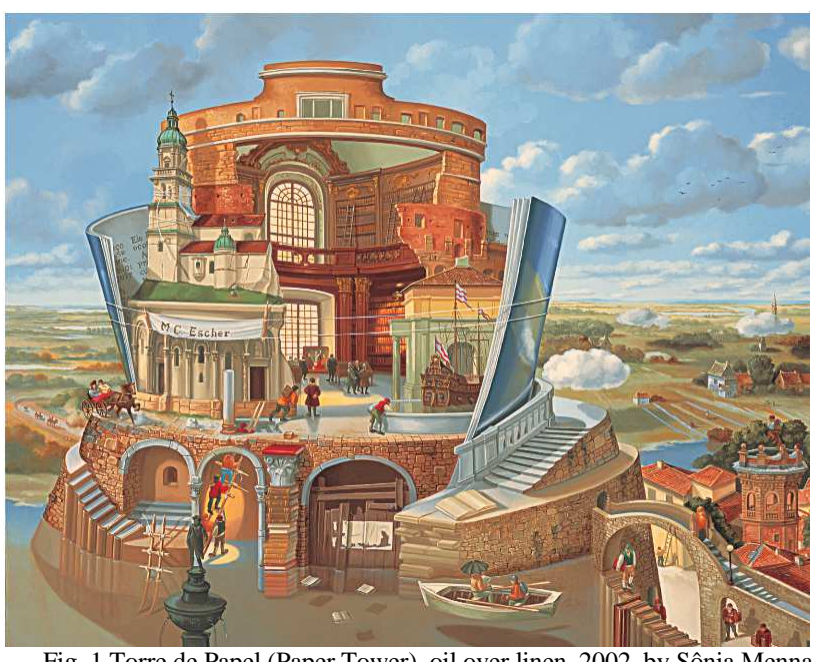

Fig. 1 Torre de Papel (Paper Tower), oil over linen, 2002, by Sônia Menna Barreto.

This paper describes the entire process of the bringing this painting into an application and the results we got: how the original painting was chosen, what was necessary to convert it into a three dimensional, animated model, how the software was created and, last but most important of all, the final application running in an immersive environment (a mini CAVE), in desktops, in digital televisions (DTV) and even in mobile platforms. We want to show that the medium used to see the artwork, though it is part of the experience and affects the perception of the user, is not a limiting factor and interactivity can be present in many forms. Just as we can appreciate a sketch of a master painter, we can also immerse 
and live in a digital artwork whether it is all around us or as small as the palm of our hands.

The concept of this project is to bring the viewer closer to the artwork, and not only be a mere spectator three feet away from it. Art is an experience, and if we can improve this experience with better tools, if we can convey more feeling and we can take the viewer into the work, art will have achieved its goal. Paintings, ordinarily static and picturing a single moment in time and space, can become dynamic, three dimensional, living entities. A whole new range of possibilities comes to mind.

Our choice of a living, contemporary artist was made by design. The artist was present during the development of the project, hence we managed to keep her idea and feelings present. Yet, when she first saw the application, she said: "it is beautiful to be able to see the backside of my painting." The chasm was bridged: the artist herself had a richer experience of her own work, was able to perceive things that she had not previously considered or at least experienced directly.

The basic idea of this project was to bring the painting into a virtual world. We wanted to make it freely explorable by the viewer-actors in first person, with virtual tours, and interacting with the work and even with each other.

This led to some requirements that had to be fulfilled:

- Immersive and interactive navigation, allowing objects and details of the painting to be explored and seen even those not visible from the original perspective of the painting;

- Animated characters. The digital work should be alive, and not merely a static 3D version of the painting;

- Realism and unusual features, such as letting the user play with the time of day, seeing the work at sunset or at noon;

- Some information about the painting itself, such as techniques, style of the artist, etc.

We took advantage of virtual reality to enhance the painting, involving technologies that let us create virtual threedimensional environments and which explore multi-sensorial devices, three-dimensional navigation, immersion in the application context and real time interaction. We will detail below the interaction, but it can be done through keyboard, mouse, WiiRemote and gestures capture by Kinect. We can also have collaborative viewing, with some people using mobile devices, others using computers and others in a CAVE.

This paper is organized as follows: section II describes the original painting chosen, "Torre de Papel" (Paper Tower) and explains how that choice was made; section III explains how the process of bringing the painting into digital art was performed, describing the technologies used; section IV presents the application itself and how users interact with it, with section $\mathrm{V}$ delving more deeply into the immersive experience in the CAVE. Section VI details the experience in the mobile and Digital TV platforms and then we end with our conclusions.

\section{THE PAINTING, TORRE DE PAPEL}

The choice of "Torre de Papel" (Figure 1) was made together with the painter. Sônia Menna Barreto's paintings, since the beginnings of her career in 1984, have been approaching the homo ludens, or the ludic man [1]. Her paintings are made using the technique of oil painting over linen, which allows even the smallest details to be painted. Her hyper-realist technique, with trompe l'oeil minutiae, (i.e. a highly detailed technique that is photo realistic), is very impressive, and her artwork is present in many private collections and even in Great Britain's Royal Family Collection.

Menna Barreto has presented her work in several single exhibits, including a large review of her work, with 44 paintings exhibited in the Museu Brasileiro de Escultura Marilisa Rathsam (MuBE-SP), in 2007.

The virtual environment presented in this paper is a perfect match to surrealism - important influence in her style - since it allows the "impossible" to be modeled and that shapes and things that are not viable in physical reality can be created and manipulated virtually.

What led us to choose "Torre de Papel" among all the possibilities of Menna Barreto's artworks are:

- Several characters and objects that are fit for exploration and animation;

- Few perspective tricks, which would be lost or impossible to keep in a real $3 \mathrm{D}$ model that could be seen from any viewpoint;

- The invisible parts of the painting, such as the back of the tower, can be completed and explored in an interesting way.

Our proposal was not just to model the painting in $3 \mathrm{D}$, converting it to an exact replica, but to complement it with elements from the artist's mythology and with multimedia content.

There is previous research and work on how to bridge the gap between traditional paintings and the digital medium. Gieseke[2] modeled Guernica, by Pablo Picasso. That project consisted in a way to visualize the dismantled figures of the city of Guernica, bombed during the Spanish Civil War. Gieseke argues that a new artwork was created, since it was something completely different from the original work due to the three dimensional exploration and how that impacted the viewer.

\section{DEVELOPMENT}

We started by studying the painting. We sketched how it would be modeled in 3D, and detailed the "invisible" parts of the painting, such as the back of the tower. Some elements of where picked from another painting of the same artist, "O lápis de Tróia" (The Trojan pencil) - Figure 2 -, helping us to fill the invisible spots. The main alteration was the replacement of the background by an ocean, thus avoiding the perspective trick of the water in the front of the tower and the altitude of the city in the back for the painting, which is not viable from other points of view. 


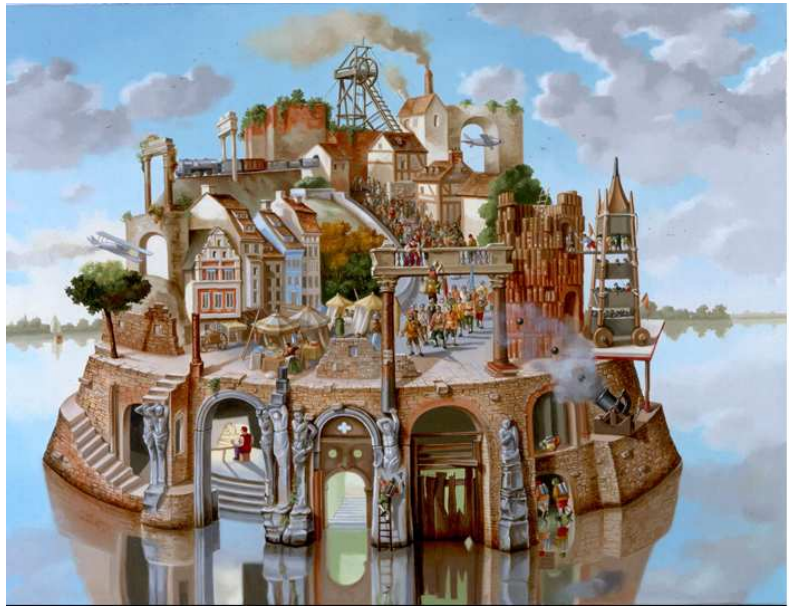

Fig. 2 "O lápis de Tróia".

We took special care to keep the textures of the model as close to the original palette as possible. Menna Barreto has a particular palette that is instantly recognizable from her paintings.

Figure 3 shows the modeled painting in 3D, in the interactive version rendered with OpenGL. The final scene has over $220 \mathrm{k}$ polygons, with about $140 \mathrm{k}$ to $220 \mathrm{k}$ visible at any given frame.

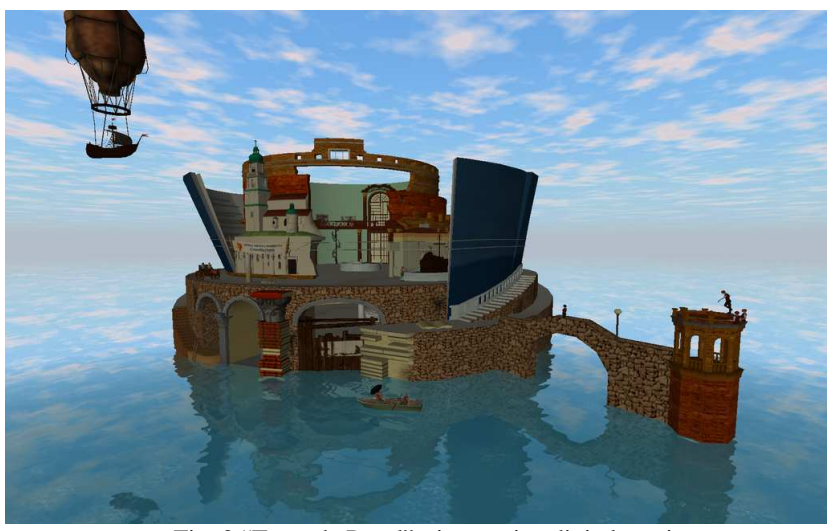

Fig. 3 "Torre de Papel” - interactive digital version.

By bringing "Torre de Papel" to a new format we wanted to explore the advantages of the digital medium. The painting has several characters, which were modeled and animated, in some cases improving the surrealist feeling. For instance, Menna Barreto suggested that the boat should not remain only on the surface of the water, but that it should fly, something that is only slightly suggested in the painting itself. Another suggestion by Menna Barreto was to add an acrobat and a painter, who are not present in the original artwork and add a meta-dimension to the digital version.

Another effect that was added to the work and which could not be done in a static canvas is the illumination. It is possible to see the painting at any time of the day, from dawn to sunset and all through the night. Figure 4 shows the tower at night.

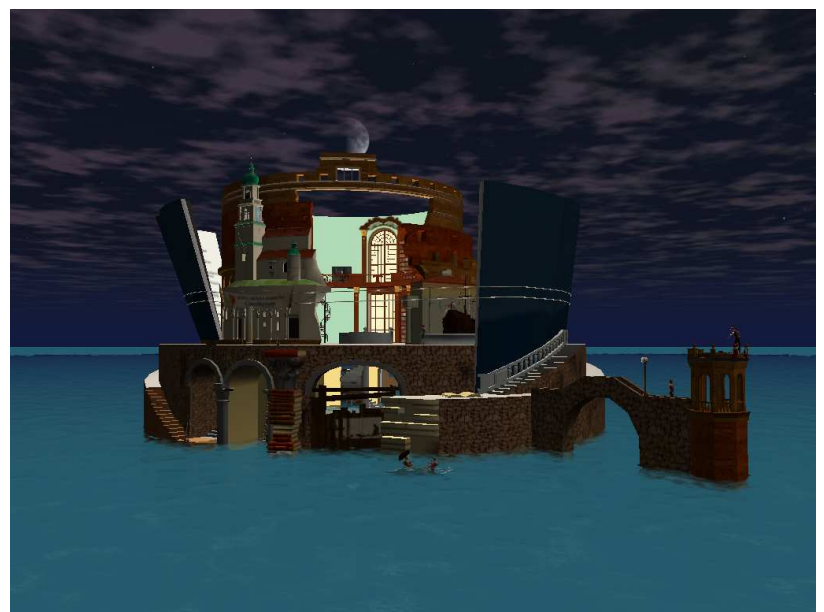

Fig. 4 "Torre de Papel" at night.

We also added an exhibit inside the tower, with many other paintings, most of them by Menna Barreto herself, and some other classic paintings that were similar in theme, such as Bruegel's The Tower of Babel. Figure 5 presents part of the interior of the tower, showing some of these paintings. The painter is animated, moving his arms convincingly.

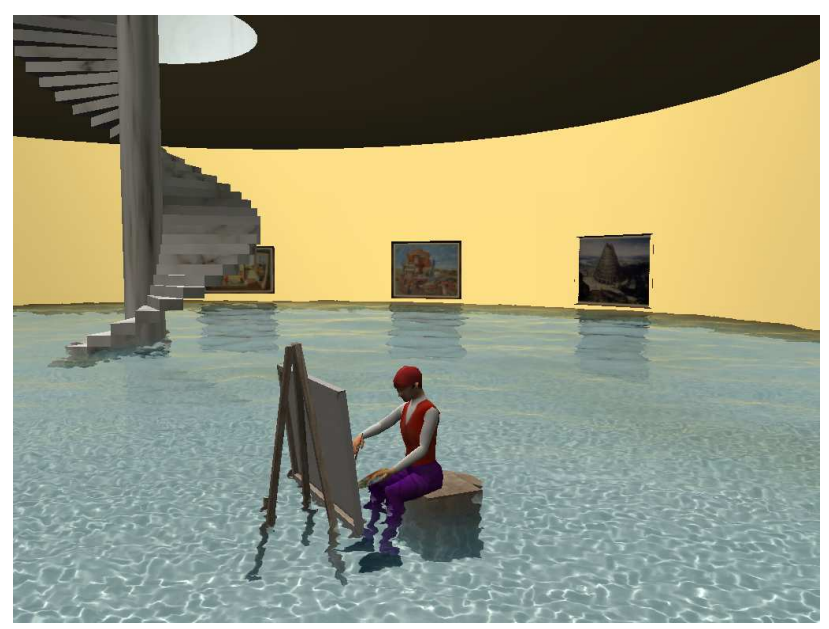

Fig. 5 Interior of the tower, something that is not visible in the original painting.

Figure 6 shows the software and hardware architecture used in the application of the digital version of "Torre de Papel". From the original artwork and extra sketches we modeled a three dimensional version, using 3D Studio Max. Some of the animations which were not interactive, such as waving flags, were already added at this step.

The model was then loaded by our own application, written from scratch. We used the OGRE3D Graphic Engine [3], and implemented the application in $\mathrm{C}++$. Audio from a soundtrack and from environment sounds (e.g. noises from the carriage) were added and localized spatially to provide a more immersive experience, using OpenAL [4], an open source library that provides multichannel and three-dimensional audio. 
Besides the traditional navigation with mouse and keyboard, we also supported two other devices, more appropriate for immersive environments. The first one is WiiRemote [5], through the WiiUseJ library[6] ; the second one is Kinect, through the OpenNI library[7].

Our application was also designed to take advantage of multiprojection environments. We used a 3-sided mini-CAVE, detailed below. We used libGlass [8] to synchronize the applications on the many nodes, a library made for distributed computing that makes the development of applications easier. This library provided all the resources we required for communication and synchronization of the cluster nodes. The solution is compatible with GNU/Linux and Windows, and Guimarães \& Gnecco [9] explain how it can be used to run virtual reality applications on distributed systems.
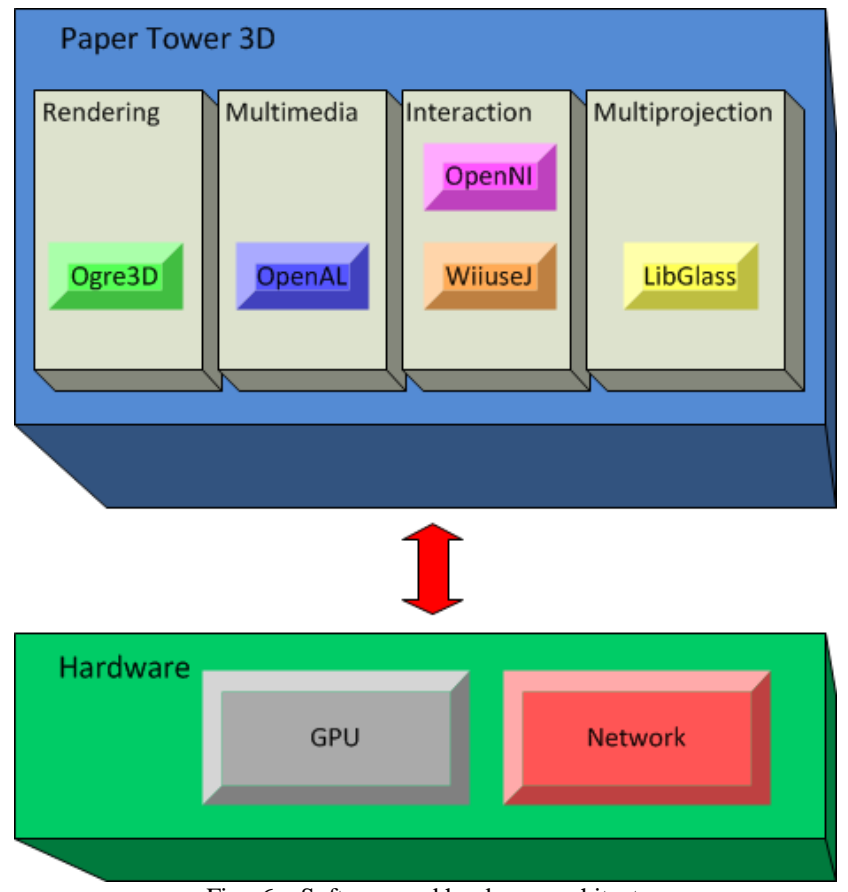

Fig. 6-Software and hardware architecture.

We implemented the usual characteristics of an interactive navigation application, such as collision detection. The ocean is rendered in real time, with a set of random waves, and uses shaders to enhance realism: reflection and refraction. Users can go under the ocean and see the tower distorted by the water refraction. The sky is also rendered in real time, with algorithms to simulate clouds (which move with wind) and the time of day: therefore sunrises and sunsets are realistic, and stars and the Moon are visible.

The application was licensed under the GNU Public License and is available at http://xptarv.sourceforge.net/. The model is licensed under the Creative Commons AttributionNonCommercial-NoDerivs 3.0 Unported License. Sônia Menna Barreto provided rights of use of the painting for this specific project.

\section{INTERACTIVITY}

Interaction is critical to the success of virtual immersive environments, since it affects directly the user satisfaction and their efficiency in performing tasks. This is because interaction affects immersion, to the point that it can generate problems known as cybersickness (headaches, nausea and postural problems) to users.

Interactivity is one of the main pillars of virtual reality applications [10]. It can be performed through conventional devices, such as mouse and keyboard, and also by nonconventional devices, such as data gloves and motion trackers. In this project we used two devices originally developed for game consoles, Wii Remote and Microsoft Kinect.

Wii Remote is a wand with motion sensors and physical buttons. Its use in navigation was deemed easy and intuitive by users, taking only a few moments to get used to. We interfaced it through the WiiuseJ library. Among the advantages of Wii Remote are its wireless interface, which uses Bluetooth to connect to the input node.

Kinect, a natural interface device, allows users to use their own bodies to generate input to "Torre de Papel". We used the OpenNI library to communicate with the device, and users could navigate around the 3D world using only their hands. Kinect cameras are connected to the input node through a USB cable. Figure 7 presents the application being run with nonconventional input devices, which are supported both in the desktop version and the CAVE version.

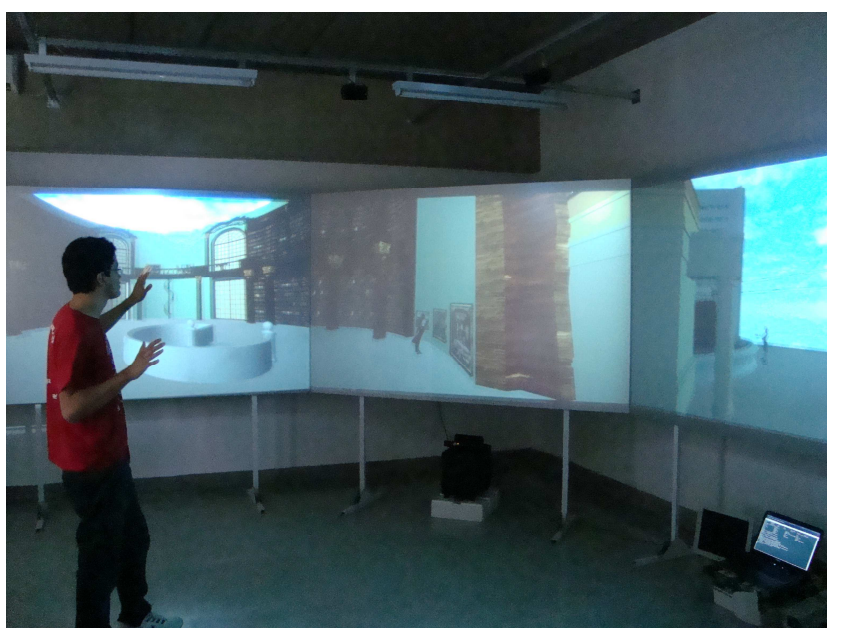

Fig. 7 - Torre de Papel 3D

\section{MINI-CAVE}

Multiprojection systems can provide a high degree of immersion to their users. The CAVE [11] was one of the first environments of this kind. The mini-CAVE used in this project was a low-budget one, with 3 sides, passive stereoscopy and six commodity PCs in a cluster. Figure 8 presents the architecture of our system. 


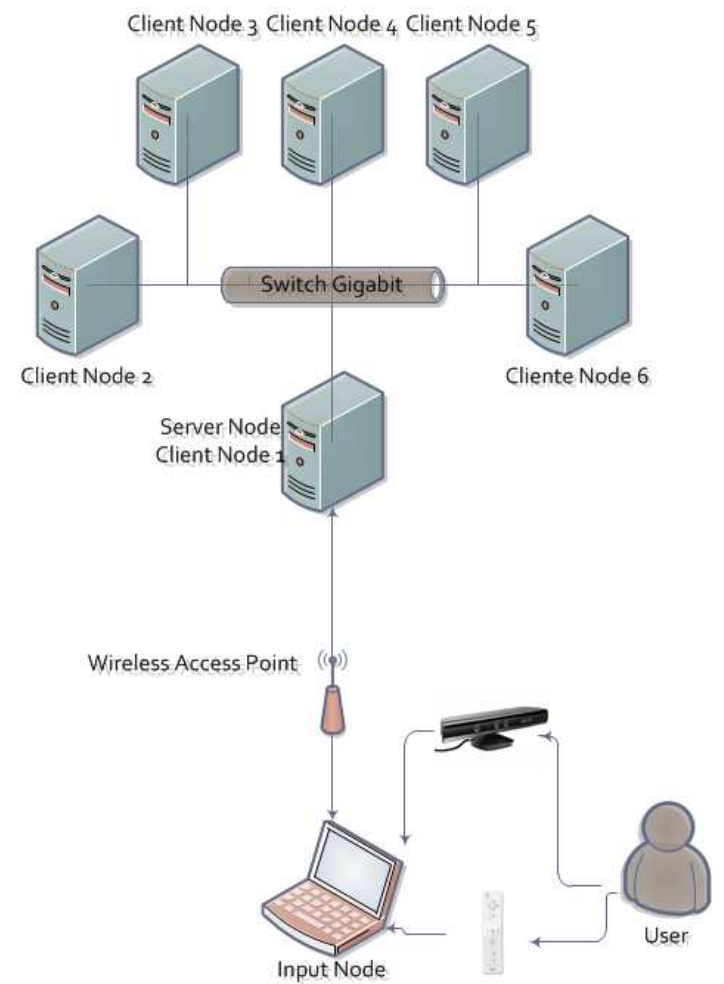

Fig. 8 - Our infrastructure for running the application in the mini-CAVE

Figure 9 presents the digital "Torre de Papel" running in a 3-sided mini-CAVE.

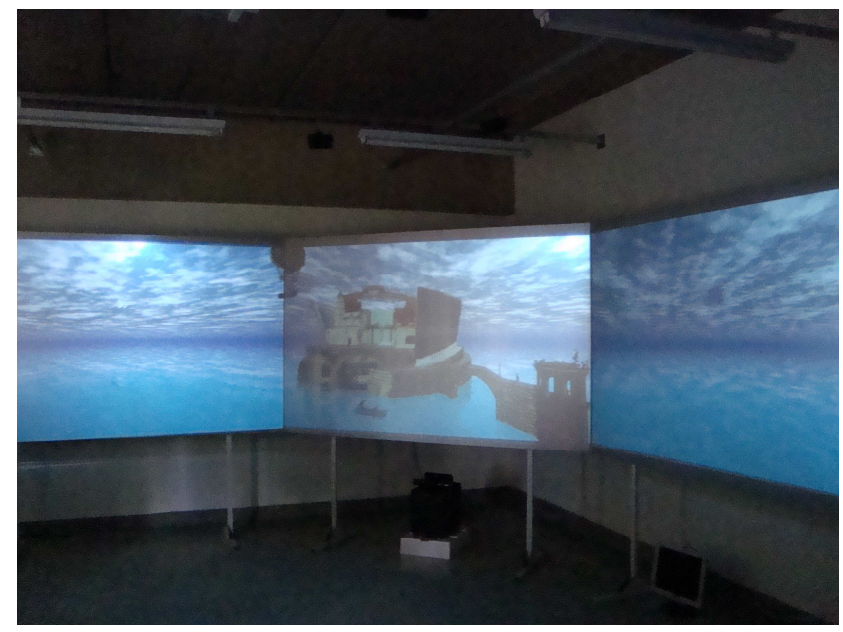

Fig. 9 - Torre de Papel 3D running in the Mini-CAVE

\section{INTEGRATION WITH INTERACTIVE DIGITAL TV}

Digital TV (DTV) systems have brought improved video and audio quality to transmissions, but have also made possible new services and possibilities, such as interactivity. They allow applications to the sent to users, on top of audio and video. When an user interacts with DTV we call it Interactive Digital TV (iDTV). Examples of interactive applications are many: electronic TV guides, games, banking services, health services, educational services, government services, and even non-linear TV shows [12].

To have interactivity in its full potential, a return channel must exist. The return channel connects the set-top box to the broadcaster, allowing the user to provide feedback on the contents being shown or even accessing the Internet. Through this channel it is possible to implement video on demand or interaction with other users.

By including several types of objects into multimedia applications we enrich the user experience, who can interact more fully and feel a greater level of immersion. Some of these objects require, however, more processing power. This is the case with 3D applications, which require hardware support for accelerated rendering, and "Torre de Papel 3D" is one of these applications. The model is relatively complex for a set-top-box, with several textures and real-time requirements: the model has to be loaded and fit into memory, as well as textures. Real-time rendering also means 30 frames per second. Hence, even though some set-top-boxes now have embedded 3D processors, their performance may not be enough for more complex $3 \mathrm{D}$ applications.

To handle this potential problem, we render the application in the PC cluster. One of the nodes renders the view in real time, transmitting the audio and video and processing the user input that comes through the return channel. As such, the actual application is not running in the set-top-box, but in a remote node. The set-top-box only acts as a proxy, decoding the audio/video stream and relaying the user input. This solution was implemented in this project [13].

After a frame is rendered in the cluster, this frame (with audio) is compressed with a real time algorithm and streamed to the set-top-box through the digital TV transmission, using a local or wide area network. The compression can be performed by a different node, if required, which can also rescale the video for different targets and resolutions. Figure 10 ilustrates this.

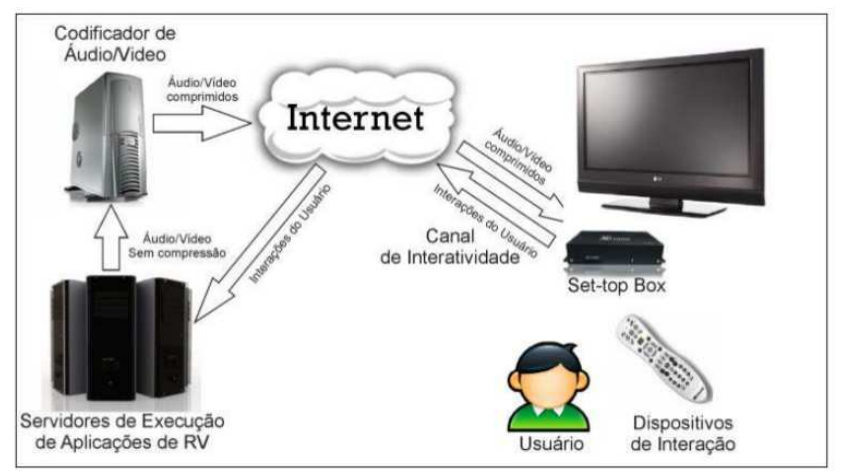

Fig. 10 - DTV Video Streaming over Internet

In figure 11 we show the actual transmission. On the left we see it transmitted to a TV, through a set-top-box. On the center we see the same transmission being sent purely over the internet to a tablet, which does the video decoding itself. Finally, on the right is the actual rendering node. We could 
render multiple views at the same time, transmitting it to different devices, but in this case the streams are synchronized. The application is being run on the CAVE at the same time, where users are interacting with it, and the cluster streams the same view to other uses who could be in remote locations.

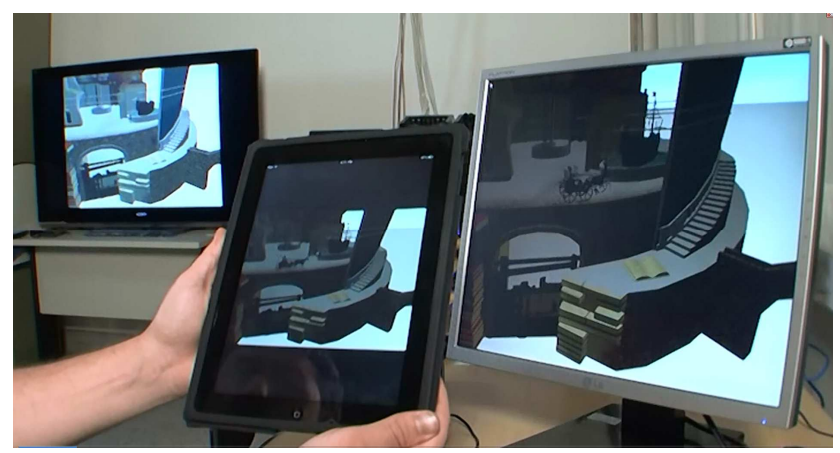

Fig. 11 - "Torre de Papel" in Digital TV (left) and tablet (center) and PC (right).

\section{CONCLUSIONS}

This research brought the painting "Torre de Papel", by the artist Sônia Menna Barreto, into a 3D digital artwork. It can be viewed in many different media: in the immersive and interactive environment of a CAVE, in desktops, in digital TVs, or in mobile devices such as tablets and smartphones. Navigation is completely interactive.

We believe this paper shows that digital art can be used to complement traditional art, bringing new forms to explore the artworks, navigating, learning about them and interacting. We did not wish to use the digital world merely to convey more information (such as informative texts), but to really immerse the viewers and take them into a whole new experience.

Art has been traditionally contemplative for several reasons, but our modern development can let the viewer interact and enjoy a richer experience. We do not see any conflicts here: paintings, sculptures and other forms of art are only benefited by technology. We had the great satisfaction of surprising the artist herself, letting her experience her own creation in a form unlike she had before.

Animation and interaction let the user immerse in the artwork, bring attention to other details that could not be noticed as easily and bring life to the pieces. We were also glad that the application could be experienced in so many different devices: digital art is often tied to a single implementation, or at least a unique platform. We do not share this view: the digital world allow us to have endless copies, all as faithful as the original yet as mutable and interactive as the users want. Bringing this project in so many devices, from multiprojection immersive environments to mobile platforms was fundamental in this sense.

We expect to extend this project with other paintings.

\section{ACKNOWLEDGMENT}

We would like to thank Sônia Menna Barreto for all the support and involvement with this project. This project was made with a grant of the Ministério da Cultura, represented by the Secretaria do Audiovisual, in partnership with the Sociedade Amigos da Cinemateca, as part of the XPTA.LAB program. We would also like to thank all researchers involved in that program.

\section{REFERENCES}

[1] A. C. Gouveia \& C. Gouveia (eds)."Sônia Menna Barreto: Pintora de Fantasias". Editora Décor, 1a edição, São Paulo, 2007.

[2] L. Gieseke. "A 3D Exploration of Picasso's Guernica". Available http://www.hdfest.com/experimental/picassosguernica.html

[3] OGRE. "Object-Oriented Graphics Rendering Engine". 2012. Available at : http://www.ogre3d.org/

[4] OpenAl. "Open Audio Library". Available at: http://connect.creativelabs.com/openal/default.aspx.

[5] NINTENDO. "Controllers at Nintendo". 2012. Available at : http://www.nintendo.com/wii/console/controllers.

[6] WIIUSEJ. "Java Api for Wiimotes: WiiUseJ”. 2012. Available at : http://code.google.com/p/wiiusej/.

[7] "OpenNI Framework". Available at: http://openni.org/

[8] M.P. Guimarães \& B. B. Gnecco, B. \& M. K. Zuffo. "Graphical Interaction Devices for Distributed Virtual Reality Systems." Virtual-Reality Continuum and its Applications in Industry VRCAI. Singapura. June. 2004.

[9] M. P. Guimarães. "Um ambiente de desenvolvimento de aplicações de Realidade Virtual baseadas em aglomerados gráficos". Doctorate thesis. Escola Politécnica da Universidade de São Paulo. Dezembro. 2004

[10] C. Kirner \& R. Siscoutto. "Realidade Virtual e Aumentada: Conceitos, Projeto e Aplicação". Petrópolis: SBC, 2007. (1, ISBN 85-7669-108-6).

[11] C. Cruz-Neira \& et al. "The cave: audio visual experience automatic virtual environment". Commun. ACM, ACM, New York, NY, USA, v. 35, p. 64-72, June 1992. ISSN 0001-0782. Available at: 〈http://doi.acm.org/10.1145/129888.129892〉.

[12] S. D. J. Barbosa \& L.F. G. Soares . 2008. "TV digital interativa no Brasil se faz com Ginga: Fundamentos, Padrões, Autoria Declarativa e Usabilidade”. Em Kowaltowski T., Breitman K. Atualização em informática 2008. Rio de Janeiro, RJ: Editora PUC-Rio. pp. 105-174.

[13] C. C. Viel \& L. C. Trevelin \& C. A. C. Teixeira \& E.L. Melo. "RV-MTV: Framework para Interação Multimodal com Aplicações de Realidade Virtual em TV Digital e Dispositivos Móveis". In: Brazilian Symposium on Multimedia and the Web (WEBMEDIA), 2011, Florianópolis. Brazilian Symposium on Multimedia and the Web (WEBMEDIA), 2011. 